\title{
FRAGMENTACIÓN URBANA Y ASOCIACIONES VECINALES EN SAN CARLOS DE BARILOCHE, PATAGONIA - ARGENTINA (1983-2015)
}

\author{
Brenda Matossian ${ }^{l}$ \\ CONICET - IMHICIHU
}

\section{RESUMEN}

Las ciudades bajo desarrollo capitalista experimentaron a lo largo de las últimas décadas profundos procesos de fragmentación urbana. Aquellas con función turística agudizaron este panorama dado el aumento del valor del suelo urbano. Tal es el caso de San Carlos de Bariloche, en la Patagonia andina argentina. Su alta compartimentación, sumada a la gran dispersión espacial de su ejido municipal y topografía accidentada, limitaron la funcionalidad y cohesión interna.

Se propone avanzar en la comprensión de dichas fracturas urbanas a partir del estudio de las asociaciones vecinales. Se trabaja desde la escala del barrio entendido como condensador de problemáticas globales, nacionales y locales y a partir de la figura de la Junta Vecinal en tanto unidad primaria de participación y principal articuladora en las relaciones entre vecinos, barrios y gobierno local. La hipótesis propone que la gestión, las relaciones y funciones asumidas desde las Juntas Vecinales, íntimamente vinculadas a la condición de clase de sus vecinos, permiten una mejor interacción a escala intraurbana y promueven situaciones más inclusivas en los sectores populares. Se da cuenta también del rol de las Juntas Vecinales en la construcción de imaginarios urbanos que inciden en un acercamiento-alejamiento de las distancias sociales intraurbanas.

Palabras clave: Desigualdad socioterritorial; fragmentación urbana; barrio; San Carlos de Bariloche; Juntas Vecinales.

\section{URBAN FRAGMENTATION AND NEIGHBORHOOD ASSOCIATIONS IN SAN DE BARILOCHE CITY, PATAGONIA - ARGENTINA (1983-2015)}

\section{ABSTRACT}

Cities under capitalist development have experienced, over recent decades, deep urban fragmentation processes. Those with a tourist function worsened this situation because of the urban land value increment. Such is the case of San Carlos de Bariloche city in Argentina's Andean Patagonia. High fragmentation combined with large spatial dispersion of its township lands and hilly landscape, have limited the functionality and inner cohesion of the city.

The aim of this article is to advance in understanding these urban fractures from a neighborhood associations study perspective. The neighborhood scale focuses global, national and local issues and the figure of the "Neighborhood Council" (Junta Vecinal) is considered as the primary unit of participation and main linkage in relations between neighbors, neighborhoods and local government. The hypothesis proposed is that the management, relationships and functions taken from the Neighborhood Councils, closely linked to its population class status, allow better interaction in the intra-urban scale and promote more inclusive positions in the popular sectors. Moreover, the role of Neighborhood Councils is relevant in the construction of urban imaginaries affecting the intra-urban social distances.

Keywords: Socioterritorial inequalities; urban fragmentation; neighborhood; San Carlos de Bariloche; Neighborhood Councils.

${ }^{1}$ CONICET- IMHICIHU. Instituto Multidisciplinario de Historia y Ciencias Humanas, Saavedra 15, Piso $5^{\circ}$, Ciudad Autónoma de Buenos Aires, CP 1083, Argentina. E-mail: bmatossian@gmail.com 


\section{INTRODUCCIÓN}

San Carlos de Bariloche es uno de los principales centros de atracción de población de la región patagónica, destino turístico de renombre nacional e internacional. Esta ciudad intermedia de 133.000 habitantes, según el censo de 2010, condensa varios elementos de complejidad: pertenece a una región fronteriza, entre Argentina y Chile, está inserta dentro un área natural protegida, el Parque Nacional Nahuel Huapi, y su economía se encuentra altamente concentrada y globalizada en torno al turismo (ABALERÓN, 1992). Núcleo urbano de jerarquía regional, al igual que otras ciudades turísticas intermedias de la Patagonia, su crecimiento demográfico, acelerado y escasamente planificado, generó profunda fragmentación urbana e intensa división social del espacio.

Desde la segunda mitad del Siglo XX hasta la actualidad, las mejoras en las comunicaciones y el impulso del turismo contribuyeron al aumento acelerado de su población. Según los registros censales, ostentó tasas de crecimiento que llegaron a duplicar las de la provincia de Río Negro por varias décadas (entre 1947 y 1980) e inclusive triplicar las del total del país durante un período aún más prolongado (entre 1947 y 1991). Este incremento demográfico vertiginoso ha producido una intensa y descontrolada expansión urbana a partir de la década del sesenta. Por entonces la superficie construida avanzó sobre las chacras de la antigua Colonia Agrícola Pastoril Nahuel Huapi creada a principios de Siglo XX en una búsqueda por aumentar la escasa presencia del Estado argentino en las áreas de frontera. Sin embargo, fue a partir de la creación del Parque Nacional Nahuel Huapi (1934) que el Estado nacional se instaló con fuerza y definió un modelo de desarrollo turístico de la mano de la Dirección de Parques Nacionales. Esta institución fue clave en este crecimiento demográfico y urbano, como también en el proceso de "argentinización" la frontera (NÚÑEZ et al., 2012). Estos elementos, junto con el aumento del parque automotor, la construcción de caminos propiciaron la expansión de Bariloche sobre topografía compleja dado su emplazamiento sobre una morena glaciaria, sumados a la escasa planificación y ordenamiento urbano dieron por resultado profundas fracturas al interior del espacio urbano (ABALERÓN, 1992; MATOSSIAN, 2012). El municipio presentaba una incapacidad funcional para brindar servicios básicos a los nuevos barrios y San Carlos de Bariloche comenzaba a perfilarse como una ciudad turística fragmentada espacialmente y con fuertes desigualdades socioterritoriales.

Hacia fines de los setenta, durante la dictadura militar representada localmente por intendente de facto Osmar Barberis, se profundizaron políticas que intentaban organizar la ciudad en base a valores estéticos y ecológicos enfocados hacia una ciudad "postal" (FUENTES et al., 2007). Así, aquellos elementos que no eran considerados parte del paisaje turístico se ubicaban hacia las márgenes de la ciudad, en sectores topográficamente más elevados.

Al mismo tiempo el ejido municipal se continuaba expandiendo y la gran dispersión espacial, alcanzó una superficie de más de 200 kilómetros cuadrados (tan extenso como la Ciudad Autónoma de Buenos Aires), hecho que ha restado funcionalidad y cohesión social. Asimismo, la polarización social y el aumento del valor del suelo urbano condicionaron de modos desiguales las estrategias de inserción urbana de distintos sectores de población. A través de esta trama de factores el espacio interior barilochense ha devenido en mosaicos sociales donde las condiciones de desigualdad socioterritorial llegan a alcanzar brechas alarmantes.

Presentado el contexto de esta ciudad, este artículo busca avanzar en la comprensión de dichas desigualdades y fracturas al interior del espacio urbano, atendiendo a la escala del barrio como condensador de problemáticas globales, nacionales y locales. Al mismo tiempo, la propuesta se concentrará en la figura de la Junta Vecinal como unidad primaria de participación y principal articuladora en las relaciones, más o menos conflictivas, entre vecinos y gobierno local y también entre los distintos barrios entre sí.

La hipótesis propone que el estudio de las relaciones y funciones asumidas desde las Juntas Vecinales, íntimamente vinculadas con la condición de clase de sus vecinos, en el marco de fragmentación y desigualdades socioterritoriales, permite una aproximación a los distintos niveles de interacción a escala intraurbana. Al mismo tiempo, los discursos construidos desde estas Juntas Vecinales puede reforzar o cuestionar las representaciones que sobre los barrios se 
FRAGMENTACIÓN URBANA Y ASOCIACIONES...

construyen en el contexto de las profundas desigualdades marcadas por un binomio de ciudad dual: la ciudad turística - la ciudad de la marginalidad.

Para dar cuenta de estos procesos se trabajó por un lado mediante el análisis de datos estadísticos obtenidos a partir de los últimos dos censos nacionales. Se procesó información a la escala con mayor nivel de detalle posible que permitiera reflejar las diferencias socioeconómicas. Luego se representaron cartográficamente las características del interior urbano también desde un enfoque material, topográfico, superpuesto con el enfoque simbólico mediante la elaboración de un mapa temático síntesis. Asimismo, mediante el uso de técnicas cualitativas, específicamente a partir de la elaboración de entrevistas en profundidad realizadas a miembros de las comisiones directivas de las Juntas Vecinales, se analizó el rol de estas asociaciones en la construcción de imaginarios urbanos que incidieran en un acercamientoalejamiento de las distancias sociales interbarriales.

\section{DESIGUALDADES SOCIOTERRITORIALES Y FRAGMENTACIÓN}

Como bien afima Clichevsky (2000:8), "el espacio es el medio a través del cual las relaciones sociales se producen y reproducen": no hay meramente divisiones sociales distribuidas en el espacio sino que la forma de la misma división social es influida por el hecho de estar localizada en el espacio (MASSEY, 1985). Es decir, que éste es condicionado y a su vez, condiciona (SANTOS, 1977). Dentro de estas nociones relacionales del espacio y la sociedad, el estudio de la ciudad se destaca como gran condensadora de procesos. A decir de Baumann (2010) la ciudad es hoy vertedero para los problemas creados y no resueltos en el espacio global. En ellas se buscan desesperadamente soluciones locales a problemas producidos por la globalización.

Existen distintos enfoques teórico-conceptuales desde donde estudiar las desigualdades socioterritoriales. Por ejemplo la noción amplia de división social del espacio urbano entendida por Duhau (2013) como la desigual distribución de distintos grupos sociales, definidos sobre todo en términos de clase o estratos sociales, pertenencia étnica, características raciales y preferencias religiosas. También refiere a la problemática otro concepto clave en los estudios urbanos como es el de segregación urbana. Este concepto ha sustentado distintos trabajos relevantes respecto a las ciudades latinoamericanas de los cuales podemos citar Clichevsky (2000), Schteingart (2001), Arriaga Luco y Rodriguez Vignoli (2003), Sabatini (2003), y Lencioni, Vidal-Koppmann, Hidalgo y Pereira (2011), por indicar apenas algunos.

Entre estos problemas propios de los estudios urbanos se destacan las desigualdades socioterritoriales y su expresión material más evidente: la fragmentación. Existen diversas maneras de concebir las formas de fragmentación urbana. Prévôt Schapira (2001:34) afirma que la fragmentación "asocia componentes espaciales (desconexiones físicas, discontinuidades morfológicas), dimensiones sociales (repliegue comunitario, lógicas exclusivas) y políticas (dispersión de actores y autonomización de dispositivos de gestión y de regulación urbana)". Por su parte las desigualdades socioterritoriales suelen ser concebidas desde una perspectiva más abarcativa. Tal como lo comprende Harvey (2007) se trata de un concepto amplio y crítico que permite dar cuenta, tanto del aumento de las brechas socioterritoriales, como de la fragmentación urbana que se produce y reproduce al interior de las ciudades.

Entre todas estas dimensiones que se articulan en estos procesos, cartografiar las diferencias según clase social de la población permite dar cuenta de cierto tipo de fracturas urbanas. Entendida la composición de clase social por tres sentidos: estructural, cultural e identificatorio (GRIMSON, 2009). El indicador medido por los censos argentinos referido a las Necesidades Básicas Insatisfechas (NBI) ${ }^{2}$ puede asimilarse al sentido estructural de dicha definición. Así, la

\footnotetext{
2 Según el Instituto Nacional de Estadísticas y Censos de la República Argentina los hogares con Necesidades Básicas Insatisfechas son aquellos que presentan al menos una de las siguientes condiciones de privación: Hacinamiento: hogares con más de tres personas por cuarto. Vivienda: hogares que habitan una vivienda de tipo inconveniente (pieza de inquilinato, vivienda precaria u otro tipo, lo que excluye casa, departamento y rancho). Condiciones sanitarias: hogares que no tienen retrete. Asistencia escolar: hogares que tienen al menos un niño en edad escolar (6 a 12 años) que no asiste a la escuela. Capacidad
} 
representación cartográfica de la distribución de la población según el porcentaje que posee NBI para dos momentos 2001 (Figura 1) y 2010 (Figura 2) permite reconocer y comparar las diferencias sociales al interior del espacio urbano barilochense. Un primer análisis del mapa resultante para 2001 (Figura 1) nos muestra la gran brecha existente entre ambos extremos de las categorías máximas y mínimas del indicador: mientras que algunos barrios como el Belgrano presenta muy bajo porcentaje de vecinos con NBI (en torno al 1,3\%), otros barrios alcanzan cifras indican que más de tres de cada cuatro vecinos tiene sus NBI, como son los casos de los barrios Unión, 2 de Abril y Nahuel Hue, hacia el sur de la ciudad.

En un segundo nivel se destaca que si bien se distingue el contraste de una mejor condición socio-habitacional en el sector norte de la ciudad, en la zona turística cercana a la costa del lago Nahuel Huapi, también se distingue que, lejos de presentarse como un todo homogéneo, el sector centro, sur y sudoeste (que componen el denominado Alto) presentan diferencias notables entre un barrio y otro. Si bien no es el objetivo de este trabajo profundizar al respecto, en trabajos anteriores (ABALERÓN, 1992, 1995; MATOSSIAN, 2010) se ha concluido que la historia de cada barrio, así como también las políticas de vivienda y planeamiento y las particularidades demográficas de sus pobladores configuran múltiples diferencias, no solamente sociales y económicas, al interior de la ciudad.

En este sentido, y frente a una tendencia general de las últimas décadas en América Latina donde el Estado "ha abdicado a su papel de garante del derecho a la vivienda, mientras que las fuerzas del Mercado no ofrecen alternativas accesibles" (OLSSON, 2012:7) es evidente que para la población con menores recursos las estrategias a desplegar con miras a integrarse y acceder a la ciudad serán significativamente más costosas y dificultosas que para el resto de la población. Como bien describe Gravano hacia 1997 el contexto de la fragmentación en las ciudades medias es de "desempleo creciente y privatización de servicios públicos y consumos colectivos que dejan a los actores de la ciudad librados a la autogestión, clientelismo e intemperie social inéditos" (GRAVANO, 1997: 3). A pesar de esta realidad, especialmente cruda en los períodos de neoliberalismo más agudo en San Carlos de Bariloche grafican el fin de la década menemista. En la última década se han implementado políticas públicas vinculadas a la tierra y la vivienda que han comenzado a mejorar, lentamente, estas profundas desigualdades socioterritoriales. Estos cambios recientes pueden visualizarse, en parte, en la Figura 2. Si bien las unidades espaciales son distintas, es posible comprobar que entre 2001 y 2010 los porcentajes máximos han disminuido de $90 \%$ a $60 \%$ y que numerosos sectores de la ciudad han mejorado los porcentajes de sus habitantes u con condiciones desfavorables.

También es interesante contraponer estos elementos con aquellos propios del emplazamiento de la ciudad, vinculados a la dimensión física de la fragmentación urbana. El interior del espacio urbano de San Carlos de Bariloche posee complejas irregularidades por su topografía, propia de una ciudad de montaña, tal como se muestra en la Figura 3 donde se representan las curvas de nivel para el sector central de la ciudad. Esta geomorfología imprime condicionamientos desde distintos planos. Desde el funcional porque genera superficies discontinuas en la ocupación por las fuertes pendientes, las cuales alcanzan rangos de entre el 30 y el $45 \%$, categorizados como "poco aptos para la urbanización" según indica el Plan Urbano Ambiental de la ciudad (MUNICIPALIDAD DE SAN CARLOS DE BARILOCHE, 2011). Desde las posibilidades de movilidad y comunicación ya que los accesos al área central y a distintos sectores suburbanos se ven dificultados por la accidentada topografía. Asimismo, las diferentes altitudes (entre $750 \mathrm{y}$ 1200 metros sobre el nivel del mar) definen condiciones climáticas más rigurosas en aquellos barrios hacia el sur, en la porción más elevada de la morena glaciaria, donde las precipitaciones invernales en forma de nieve acusan una intensidad significativamente mayor a la que se registra en los sectores cercanos a la costa del lago. Entonces, la topografía constituye un elemento significativo que imprime condiciones más o menos favorables a los vecinos que habitan estos espacios. Así cobra protagonismo en los procesos de fragmentación urbana. A esto se suman los elementos que emergen desde el plano simbólico que contrapone un sector

de subsistencia: hogares que tienen cuatro o más personas por miembro ocupado, cuyo jefe no hubiese completado el tercer grado de escolaridad primaria.

(http://www.indec.gov.ar/glosario/textos_glosario.asp?id=21) 
FRAGMENTACIÓN URBANA Y ASOCIACIONES...

conocido como el "Alto", con una directa connotación negativa vinculada a la pobreza y marginalidad, de otro cercano al lago Nahuel Huapi; la ciudad "para el turismo". Estas representaciones serán desarrolladas con mayor detalle en el próximo apartado.

\section{FIGURA 1}

San Carlos de Bariloche, barrios. Población con Necesidades Básicas Insatisfechas 2001

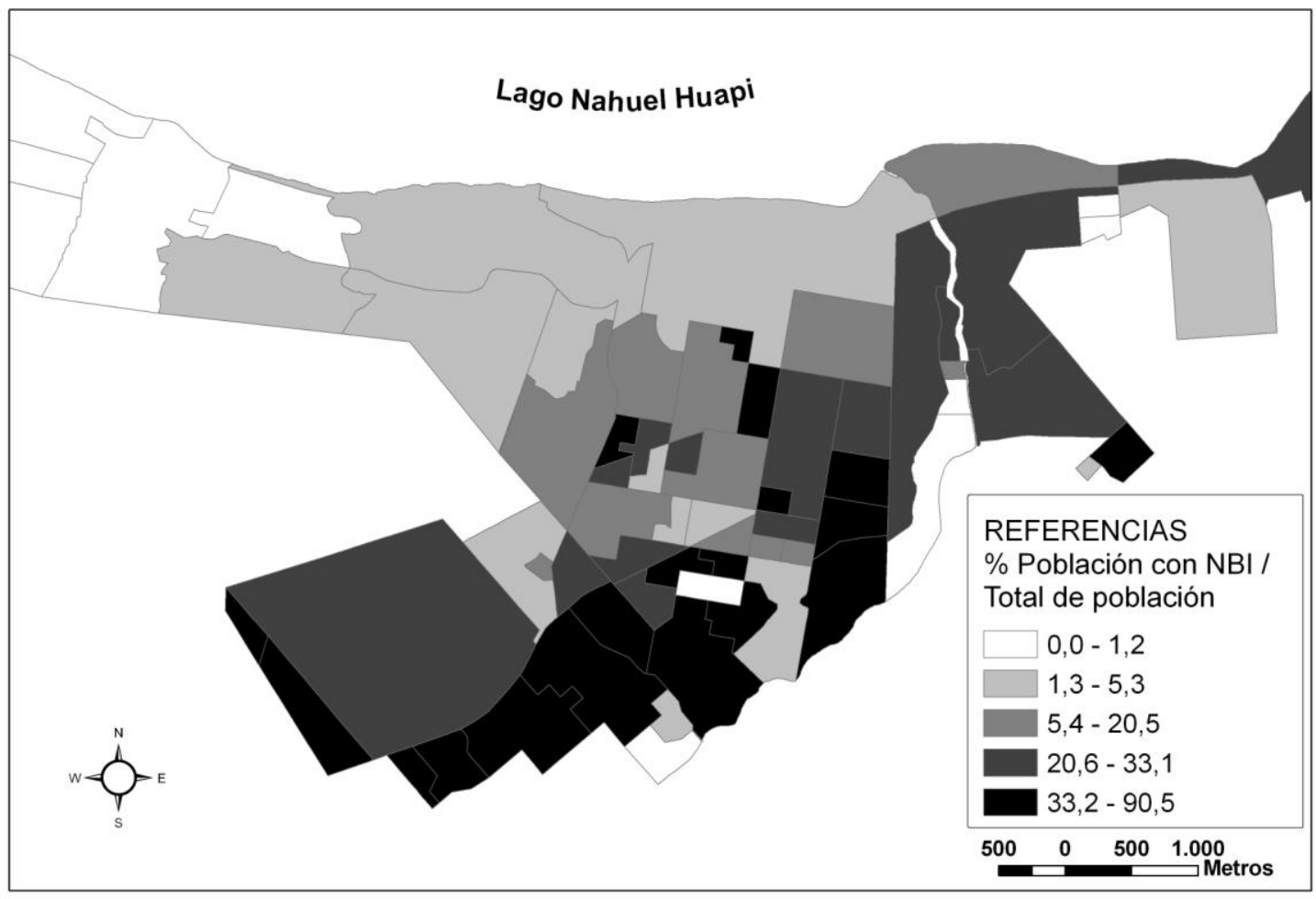

Fuente: Elaboración propia sobre la base de datos de la DGEC, 2005

FIGURA 2

San Carlos de Bariloche, radios censales. Población con Necesidades Básicas Insatisfechas 2010

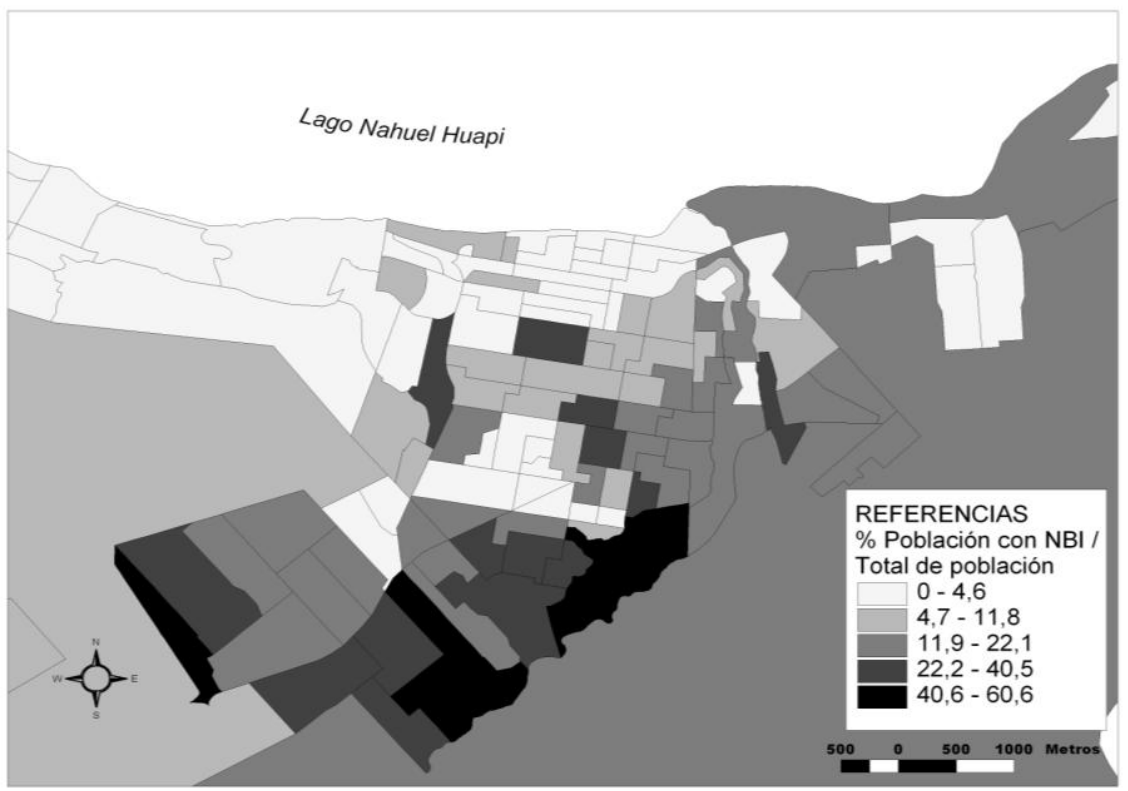

Fuente: Elaboración propia sobre la base de datos del Censo Nacional de Población, Hogares y Viviendas, 2010 


\section{LA DIMENSIÓN SIMBÓLICA DEL ESPACIO INTERIOR}

En un contexto de décadas de escasa intervención estatal en la construcción de infraestructuras, equipamiento urbano, de servicios y fuerte impacto del mercado inmobiliario sobre la renta del suelo urbano (MEDINA, 2013), se ha instalado ya hace más de medio siglo la idea de las dos caras de San Carlos de Bariloche. Se trata de un modelo de ciudad dual constituido por una reducción binaria: una ciudad inmersa en el bello paisaje de lagos y montañas, destinada al turismo y a las clases acomodadas y la otra, la de la pobreza y la exclusión en sectores urbanos vulnerables, la que no mira al lago ni accede al paisaje valorado turísticamente, reconocida como "El Alto" (Figura 3). Estas representaciones continúan sedimentadas en el sentido común que reproduce las desigualdades, las naturaliza y desconoce las complejidades al interior de los sectores aparentemente homogéneos. Lejos de tal concepción binaria, la ciudad presenta una compleja y heterogénea composición social y espacial. En la Figura 3 además de estas "dos caras" se reconoce también el área de edificios planificados por el Instituto Provincial para la Promoción de la Vivienda de Río Negro (IPPV). Este espacio otorga un paisaje muy particular a ese sector de la ciudad en un borde urbano de altas pendientes, correspondiente a la cota de los 850 metros. Al mismo tiempo, entre la "ciudad para el turismo" y el sector de edificios del IPPV se propone el reconocimiento de toda una gran franja que cuestiona una vez más estas "dos caras" a partir de la identificación de un "ecotono", en tanto metáfora de transición entre distintos paisajes. Se constituye como un amplio sector de asentamiento propio de clases medias. Lamentablemente, en los imaginarios y las prácticas urbanas muchas veces las figuras que se mantienen con fuerza semejan más a las de una barrera que a la de una zona de transición. La Figura 3 propone esta relación entre las representaciones y la topografía local como modo de comenzar a desarticular estas construcciones simplificadoras.

FIGURA 3

Topografía y representaciones sobre el espacio en San Carlos de Bariloche

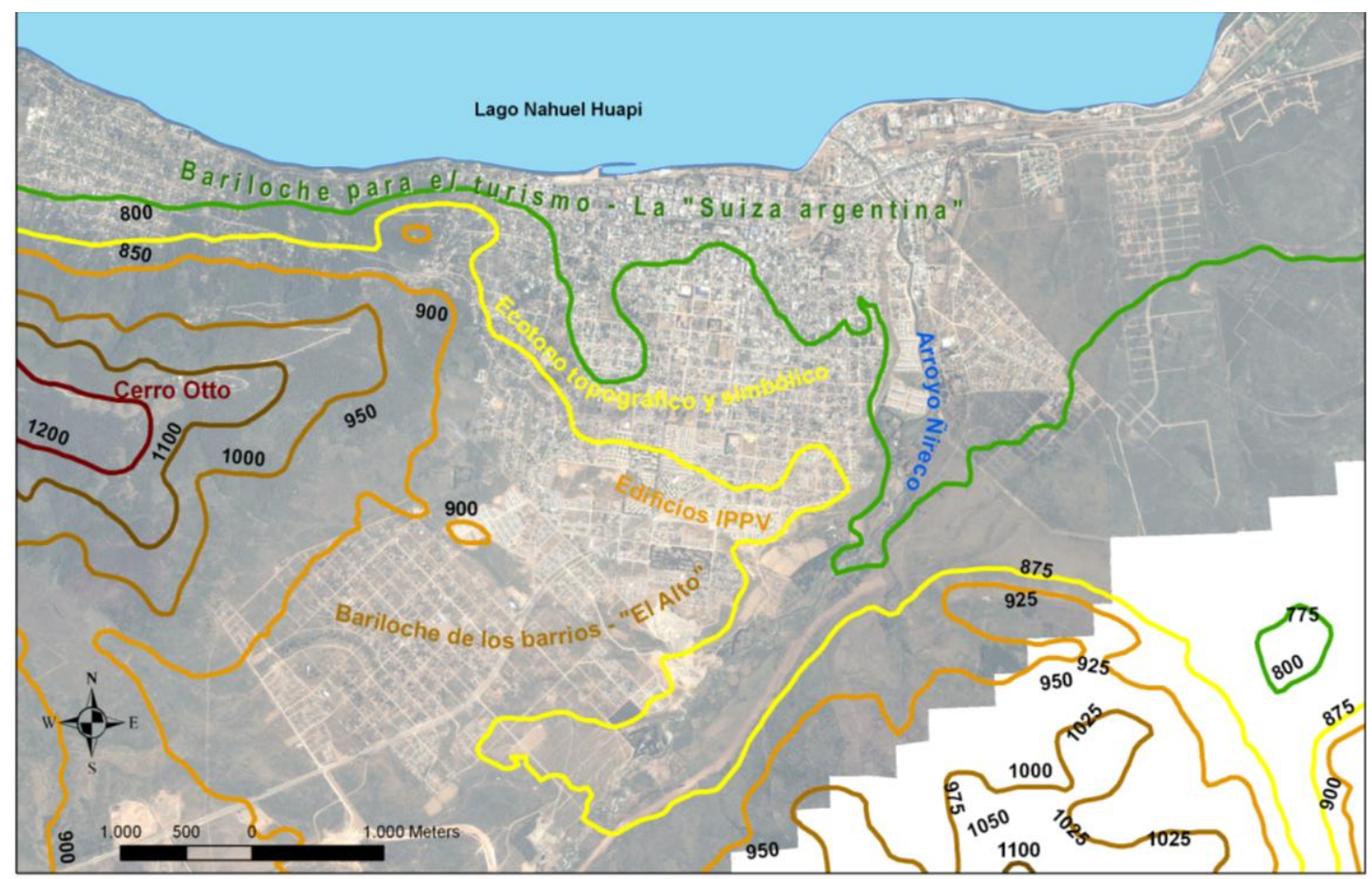

Fuente: Elaboración personal

Cabe agregar también que estas representaciones cristalizan las fuertes tensiones entorno a la construcción del pasado barilochense y el relato hegemónico de sus primeros pobladores. Los conflictos entre las "varias historias" tienen un trasfondo étnico-cultural latente anclado en la 
idea de Bariloche como una "Suiza argentina", edificada a finales de la "Campaña del Desierto" (NAVARRO et al., 2009). Con la "Campaña del Desierto", que en el sector del Nahuel Huapi se concretó entre 1881 y 1885 , el ejército argentino controló las tierras indígenas, asesinó y desplazó a distintos grupos que habitaban la región para incorporar esas tierras a la lógica económica dominante promotora de un poblamiento blanco y europeo (MÉNDEZ et al., 2001; KROPFF, 2001; NÚÑEZ, 2004, MÉNDEZ, 2005; FUENTES et al., 2007; NAVARRO FLORIA, 2008; MATOSSIAN, 2010). Desde esta idea se buscaba un poblador "ideal", "blanco y europeo" para conformar la "Suiza argentina". Dado que el poblamiento tiene sus propias dinámicas, difícilmente controlables por el Estado nacional, la movilidad humana se expandió y avanzó más allá de las pretensiones homogeneizadoras y sus fronteras. Se conformó así, a lo largo de su historia como ciudad, una sociedad diversa y compleja.

La identificación de esta sociedad con las migraciones se ha construido a partir de esta idea de en la que un conjunto de pioneros centroeuropeos forjaron la colonia agrícola pastoril a principios del Siglo XX. Este relato se ha cristalizado principalmente a partir de la única actividad directamente vinculada con la presencia de extranjeros legitimada y reconocida durante décadas: la Fiesta de las Colectividades Europeo-Argentinas. Esta fiesta reúne agrupaciones que representan a España, Austria, Alemania, País Vasco, Suiza, Rusia, Eslovenia, Croacia, Italia y Dinamarca. Esta actividad goza de prestigio y buena prensa y se sostiene al día de hoy como la expresión más fuerte del "buen migrante" a nivel local. En los últimos veinte años existieron períodos en los cuales las "otras" migraciones cobraron cierto protagonismo, mediante distintos procesos, algunos en principio excluyentes y otros, más recientes, afortunadamente inclusivos.

Sin embargo, este antecedente, da cuenta de la continuidad de un fuerte elemento de tensión en la presencia "problemática" de ciertos grupos vistos como ajenos, vecinos "menos legítimos" asentados en barrios que se buscó excluir del mapa. Los "otros" para el relato hegemónico de la "Suiza argentina" refirió a las comunidades indígenas, el grupo mapuche principalmente, junto con migrantes internos de la empobrecida Línea Sur de Río Negro y migrantes internacionales, mayormente chilenos (KROPFF, 2001; FUENTES et al., 2007; MATOSSIAN, 2010) a los que se agregaron más recientemente otros migrantes laborales de países de América del Sur (bolivianos, paraguayos y colombianos). La condición de clase atraviesa a la conformación de estos heterogéneos conjuntos, da cuenta de procesos desiguales de legitimidad ciudadana y posibilidades diferenciales de participación. Así, tal como indica Celia Guevara se trata de nuevas formas de problemas fronterizos, con una clara perspectiva de clase (GUEVARA, 2014).

\section{CIUDAD Y SUS FRAGMENTOS: LA IMPORTANCIA POLÍTICA DEL BARRIO}

Frente a la noción de ciudad en tanto espacio de encuentro entre diferentes, Baumann refiere a una batalla entre los dos extremos del comportamiento humano (cuando se presenta la opción por mezclarse con otro diferente) bajo los términos de "mixofilia y mixofobia"; al fin, dice, "todo recae sobre la población local, sobre la ciudad, sobre el barrio" (BAUMAN, 2010). Y he aquí uno de los centros de interés: el barrio, que no debe ser considerado una mera división jurisdiccional, administrativa o física, es también una formación histórica y cultural, de importancia material y simbólica para todos sus habitantes. A ello se agrega su papel estratégico ante los procesos de fragmentación urbana, las múltiples dimensiones de la desigualdad socioespacial y su rol como unidad jurisdiccional en los mecanismos de participación ciudadana.

Desde esta perspectiva multidimensional y multiescalar, de lo global al barrio, donde los conflictos propios de la vida urbana se entrelazan con los problemas de la diversidad cultural, se busca contribuir al conocimiento de las dimensiones políticas de la desigual inserción de los vecinos y su derecho a la ciudad. Se entiende el derecho a la ciudad, como el pleno ejercicio de la ciudadanía de sus habitantes, vinculado íntimamente con un tipo de gestión democrática. Este derecho es expresado en la participación de la sociedad de forma directa y participativa en el planeamiento y gobierno de las ciudades.

La importancia de los barrios en las ciudades ha interesado a distintos estudiosos de las Ciencias Sociales desde hace ya un largo tiempo, en particular desde la Escuela de Chicago y 
las teorías clásicas urbanas. Más cercanos en el tiempo se destacan, desde la geografía humana francesa, los aportes que vinculan la escala del barrio y la inserción de lo migrantes de Grafmeyer (1998) y Baby-Collin (2005). Localmente, existen notables contribuciones recientes en la línea de los estudios urbanos como los de Gravano (2005), Merklen (2005) y Pavcovich (2011).

El barrio ha sido analizado también en tanto unidad de distinción dentro del espacio urbano respecto a la diferencial renta del suelo urbano de la ciudad capitalista (TOPALOV, 1984). Además ha sido estudiado desde variadas perspectivas subjetivas, en particular por la antropología urbana. Asimismo, desde organismos internacionales como el Council of Europe (1998) se destaca la importancia de comprender las necesidades y realidades culturales de los barrios y de advertir a los planificadores urbanos y a los tomadores de decisiones sobre políticas urbanas sobre su relevancia. Se conciben los barrios como oportunidades de expresión de los locales, como lugares de miedo pero también de esperanza como lugar de intercambio y encuentro con el otro, de democracia y de participación, creación e innovación, lugares de contradicción de nuestra era. El barrio es visto como un asunto político y social, donde se construyen identidades, un lugar en el cual la comunidad busca darle un sentido a todo un compendio de prácticas culturales y modos de vida (COUNCIL OF EUROPE, 1998: 6).

Para el caso de San Carlos de Bariloche existen distintos antecedentes de trabajos anclados en esta escala (KROPFF, 2001; FUENTES et al., 2007; MATOSSIAN, 2012; AGÜERO, 2008). En estos trabajos, dedicados al estudio de barrios populares, se da cuenta de los fuertes rasgos de exclusión y estigmatización de los habitantes que en ellos residen. Estos barrios comparten algunas características pero se distinguen en muchas otras.

El análisis del derecho a la ciudad desde los barrios implica reconocer estos espacios como posibilidades de despliegue un poder colectivo que busca participar en las decisiones para remodelar los procesos de urbanización a pesar de las relaciones asimétricas existentes y la legitimidad diferencial entre vecinos de la misma ciudad.

\section{ASOCIACIONES BARRIALES: LAS JUNTAS VECINALES COMO VEHICULIZADORAS DE LA PARTICIPACIÓN CIUDADANA}

En la escala local, los mecanismos y estilos de participación ciudadana, en diálogo con el gobierno municipal, dan cuenta de las tensiones presentes en la construcción de legitimidades entre los vecinos, según clase social, a su vez, se presenta como una ocasión para atenuar o bien reproducir las desigualdades socioterrioriales.

Las Juntas Vecinales (JV) son reconocidas en Río Negro a través del artículo $\mathrm{N}^{\circ} 240$ de la Constitución Provincial que indica que: "los municipios y comunas reconocen la existencia de las juntas vecinales electivas. Se integran para promover el progreso y desarrollo de las condiciones de vida de los habitantes y sus vecindarios. Las autoridades de las juntas vecinales tienen derecho a participar con voz en las sesiones de los cuerpos deliberativos únicamente en los problemas que les incumben en forma directa. Pueden administrar y controlar toda obra o actividad municipal que se realiza en la esfera de sus delimitaciones vecinales, en colaboración $y$ dependencia con los gobiernos municipales y comunales, de acuerdo a las reglamentaciones". La Carta Orgánica las incluye en su artículo $\mathrm{N}^{\circ} 165$ donde indica que la Municipalidad reconoce y fomenta la creación de JVs para "la satisfacción de necesidades comunes, mediante toda modalidad de participación comunitaria".

El primer antecedente importante, desde la esfera municipal, fue la Resolución del Consejo Municipal N 37 del año 1958 (posterior a la provincialización de Río Negro en 1955) donde se aprobó la creación de las JVs Provisorias. Sin embargo, su reglamentación no se hizo efectiva hasta el retorno de la democracia en 1983 cuando fueron reconocidas, en el año 1986, mediante la Ordenanza 194-CM-86 en la cual se instituye el Reglamento de JVs. Allí se indica que se formarán como asociaciones civiles simples, y su reconocimiento les otorgará personería jurídica municipal.

Desde un enfoque de las dimensiones socioeconómicas, la importancia de la JV es muy relevante para la vida cotidiana de los vecinos en los sectores populares (FUENTES et al., 2007; MATOSSIAN, 2010). Además de ser articuladora de gestiones y reclamos materiales de los 
FRAGMENTACIÓN URBANA Y ASOCIACIONES...

vecinos frente el estado municipal, ostenta un papel como condensadora de intereses y luchas simbólicas y como institución con la cual se establecen fuertes lazos de identificación. Gracias al trabajo desde las JVs los vecinos han podido mejorar las condiciones de profunda desigualdad con las que nacieron muchos de los barrios del "Alto" tal como recordaba un miembro de la Comisión Directiva de la JV del barrio San Francisco II y III: "compré el terreno en 1980, no había nadie por acá, no había calles, eran huellas y no había ningún servicio, nada, ni luz".

En el barrio Frutillar, en el año 1988 los miembros de la JV lograron la conexión del barrio a la red de agua potable (Ordenanza $094-\mathrm{C}-88$ ), también la donación de un lote por parte del Concejo Municipal para la construcción de la escuela del barrio. En el barrio Arrayanes las gestiones permitieron la instalación de un campo deportivo (Ordenanza 891-94), la construcción de una sede social, sala de primeros auxilios y la incorporación de una línea de transporte urbano de pasajeros (Ordenanza 127-I-82). La reconstrucción de las historias de gestión barrial a través de las JVs da cuenta de los avances que estas instituciones han logrado en la calidad de vida de los vecinos y en el fortalecimiento de una identidad barrial y un sentido de pertenencia local. Este rol se sostiene en el tiempo y ha acompañado la expansión urbana hacia las nuevas periferias urbanas, con la creación de nuevos barrios, de origen más reciente. Tal es el caso del barrio Nahuel Hue, cuya presidenta relata "en el 2008 empezaron las elecciones asi que ahí empezamos entonces a armar la Junta ... a trabajar, empezamos con reuniones, convocatorias y empezamos a luchar por el tema de los terrenos, después por el tema del agua, de la luz... o sea este barrio no tenía posteado de luz, no tenia agua, nada... es un barrio que se creó hace muy poco y tiene todo... porque tenés la red de agua gratis, el gas viene la red troncal gratis, es un barrio que avanzó muchísimo".

En contrapartida, se visualizan cambios en un sentido divergente en barrios con una realidad socioeconómica muy distinta. Tal es el caso de Villa Arelauquen. La JV Villa Arelauquen fue reconocida en 1986 y se le otorgó Personería Jurídica Municipal en la Ordenanza 56-E-87. Luego, con la construcción del emprendimiento privado Villa Arelauquen se convirtió toda la superficie que iba a contener el barrio en una urbanización cerrada, destinada tanto a funciones residenciales como turísticas. Hacia 2008, la Ordenanza N $^{\circ}$ 1847-CM-08 disuelve la JV Villa Arelauquen y da de baja su personería jurídica municipal. En los fundamentos se afirma que los vecinos reunidos en asamblea consideraron que no era necesaria la continuidad de la JV y solicitaban su disolución: "dado que el predio ha quedado dentro del country con todo lo que ello implica. Esto [la disolución] no genera ningún cambio para los vecinos ni para los asociados, teniendo en cuenta que la empresa Burco se hace responsable de brindarle todos los servicios que correspondan".

Para el caso del barrio Belgrano el presidente de JV definía: "Este barrio es un barrio en general de clase media donde una de las características es que hubo muchísimos alemanes viviendo en este barrio, todavia hay descendientes de alemanes y alemanes... y digamos económicamente bueno clase media... un barrio que se ha caracterizado por hacer todos los servicios el mismo barrio, es decir prácticamente todas las calles pavimentadas fueron solventadas por los vecinos, las cloacas también, el agua también, es decir el nivel económico que le permitió de algún modo eso"

Estos barrios ilustran, tal como afirma Harvey, los intereses privados o cuasi privados vinculados a una élite política y económica que interviene de una manera abrupta en el ejercicio del derecho a la ciudad. En el caso extremo de Arelauquen se llegan a considerar "innecesarios" los mecanismos de participación vecinal. De este modo se evidencia de qué modo se articulan las desigualdades socioeconómicas de los vecinos en la conformación y desarrollo de las JVs.

La Figura 4 se propone como una pequeña síntesis y muestra de los discursos que se (re)producen y contraponen desde estos ámbitos de participación según las distintas áreas de la ciudad, sus formas de concebir la participación ciudadana, las vinculaciones entre actores públicos y privados y el nivel de compromiso asumido por los actores involucrados.

\footnotetext{
${ }^{3}$ El Grupo Burco es la empresa de origen belga que ha realizado en emprendimiento inmobiliario.
} 
FIGURA 4

Juntas Vecinales: representaciones y formas de participación

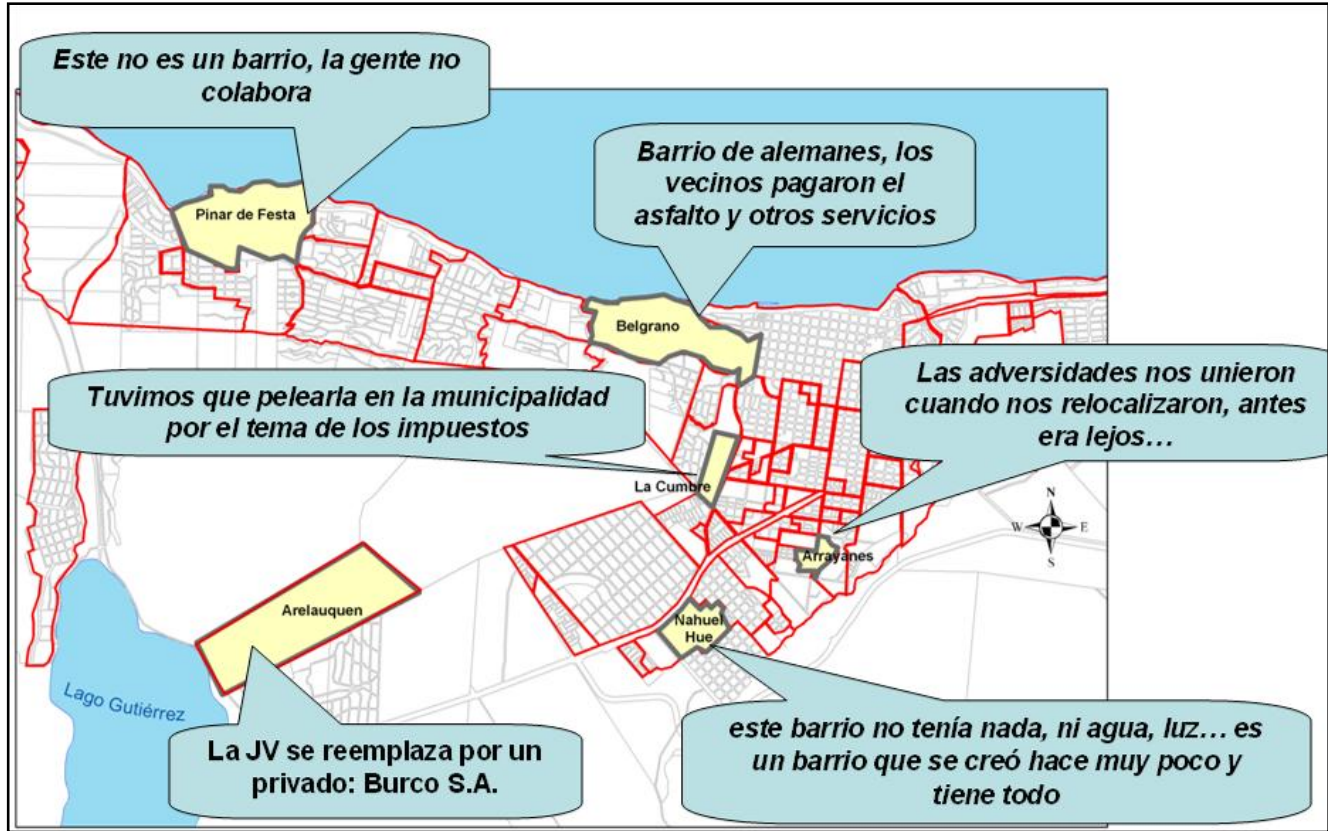

Fuente: Elaboración personal sobre la base de información obtenida en entrevistas en profundidad a miembros de Comisiones Directivas de JVs e informantes clave

\section{RELACIONES ENTRE JUNTAS VECINALES Y LA FIGURA DEL GOBIERNO LOCAL}

Otro eje analítico para comprender el impacto de las JVs en las dinámicas socioterritoriales de la ciudad, tanto desde su labor en la gestión barrial como en la construcción de representaciones, es el de la existencia, o no, de relaciones inter-juntas. Así, se indagó en el trabajo colectivo, bien para reconocer si se articulan entre sí estas asociaciones con miras a fortalecer sus reclamos frente al municipio, o por el contrario, si no se tienden redes entre JVs. También se sondearon las áreas del gobierno local a través de las cuales se puede llegar a concretar estar interacción.

Dentro del marco institucional de funcionamiento de la Municipalidad de San Carlos de Bariloche los mecanismos de descentralización y alcances previstos para la participación ciudadana se estructuran de una forma particular. La ciudad se divide en 5 Delegaciones Municipales y 9 Centros de Atención y Articulación Territorial (CAAT). Las Delegaciones Municipales cubren la totalidad del ejido municipal y cumplen una función de descentralización administrativa y tributaria, esto limita la interacción frente a otras posibles demandas que pudieran presentarse. En contrapartida los CAAT se distribuyen únicamente en ciertos sectores de la ciudad, definidos por el criterio de atención a familias de mayor riesgo social. Según la misma municipalidad es a través de los CAAT que se desarrolla el trabajo comunitario, en articulación con las organizaciones de base y a través de las Mesas de Concertación Barrial. En la experiencia de los dirigentes de las JVs se cristalizan las funciones de ambas instituciones.

Para el caso del barrio Belgrano las interacciones urbanas y de encuentro con otras JVs se da en ciertas reuniones municipales donde sienten seriamente cuestionada la legitimidad de sus reclamos: si... (nos corresponde) Delegación Centro, pero de todos modos también cuando vamos a juntas vecinales... uno se siente muy pero muy incómodo porque cuando habla de algún problema por ejemplo te dicen "ustedes que son... están en un nivel ahí jamás entienden nuestros problemas" y es como si uno no entendiera los problemas del otro y nuestros problemas fueran problemas superfluos dentro de la sociedad... hablo de seguridad, "bueno si pero ustedes no saben lo que es inseguridad..." hablo del problema de las plazas "no, pero eso no es problema..." que en algunos casos las plazas las están usando 'ocupas' así que ya... pensar tener en la plaza los árboles cortados parece ser un lujo sibarítico". 
FRAGMENTACIÓN URBANA Y ASOCIACIONES...

La presiente de la JV Nahuel Hue relata una situación muy diferente: "nos juntamos en una mesa del CAAT 8 todos los lunes y evaluamos todas las cosas, si igualmente este barrio es muy carenciado por la situación de la gente y la gente recibe una bolsa de alimentos cada 15 días que se le da a través de la municipalidad acá, la traemos acá". En una sintonía colaboracionista similar la presidente de la JV 28 de abril describe "trabajando a veces cosas en conjunto con los demás dirigentes como por ejemplo que nosotros trabajamos mucho en conjunto con los barrios, todos, todos los de acá arriba: Unión, Pilar II, 258, Malvinas, Omega, Frutillar, Quimey Hue, Progreso, Arrayanes, Vivero... funcionamos como un bloque.... Así es como conseguimos por ejemplo con una levantada de firmas conseguimos una tierra de una escuela que se va a construir... y después nosotras como... viste que nosotras las juntas vecinales nos organizamos por CAAT... nosotros estamos en el CAAT 7"

Estos dos relatos muestran elementos de interés dentro de las posibilidades de interacción JV-Municipio. Por un lado, los CAAT son protagónicos en las dinámicas y luchas por mejorar las condiciones de los barrios populares, los cuales tienen tendencia al agrupamiento frente a problemáticas puntuales tanto de forma auto-convocada como participando en instancias puestas a disposición por el mismo municipio. Simultáneamente, en los barrios más favorecidos no existe un nexo directo entre el barrio y la municipalidad.

\section{REFLEXIONES FINALES}

La ciudad patagónica de San Carlos de Bariloche ostenta fracturas urbanas, de mayor o menos profundidad, desplegadas sobre un contexto de desigualdad socioterritorial agudo de larga data. La complejidad de este panorama, propio de las ciudades bajo desarrollo capitalista, se agudiza por su condición de ciudad turística, su topografía accidentada, que tiende a reforzar las asimetrías, y las representaciones vinculadas al componente demográfico sobre las cuales se construyen imágenes de grupos de población más o menos "legítimas" respecto a su origen. Estas particularidades, combinadas con las desiguales condiciones de clase, marcan los fragmentos urbanos de modos dramáticos. Este artículo ha intentado reflejar los avances en la comprensión de las relaciones entre los procesos de desigualdad socioterritorial y las dinámicas de participación ciudadana al interior del espacio urbano barilochense.

Esta primera aproximación muestra la existencia de circuitos de participación e interacción entre espacios e instituciones centrales (dependientes del municipio) y los barrios, vehiculizadas por el trabajo de los CAAT que se concreta en las Mesas de Concentración. Estas relaciones hacen posible la concreción en el territorio de las políticas inclusivas implementadas en los últimos años y el tratamiento colectivo de problemáticas sociales que afectaban a gran cantidad de barrios hasta hace pocos años y que lenta y parcialmente han ido mejorando. También se destaca que frente a reclamos puntuales, especialmente los vinculados con la obra pública, la gestión es más directa entre la JV y el Municipio.

Estas interacciones entre distintas esferas institucionales posibilitan además una vinculación entre áreas aparentemente distantes en el nivel de las representaciones. Continuar permeando estos muros urbanos elevados para reforzar la idea de las dos caras de la ciudad constituye una situación superadora hacia un "ecotono" que continúe la ardua tarea de desarticulación de la mirada homogeneizadora. El desafío depende de estas instituciones, de la voluntad política del gobierno local de turno y de la capacidad de gestión política y compromiso de los líderes barriales. Este último elemento muestra no sólo una evidente relación política-partidaria, sino también un riesgo de dependencia del devenir de los barrios atado a una figura individual con liderazgo.

Finalmente, el desafío que debe continuar creciendo es también el de la transformación cultural hacia una San Carlos de Bariloche más inclusiva. 


\section{BIBLIOGRAFÍA}

ABALERÓN, C. A. (1995): EURE. "Difusión espacial de la pobreza y destrucción del patrimonio natural: las dos caras de una misma moneda", vol. XXI, n 64, p. 61-74.

ABALERÓN, C. A. (1992): Tendencias de Crecimiento Poblacional y Espacial en San Carlos de Bariloche con Énfasis en el Sector Marginal. Fundación Bariloche, San Carlos de Bariloche, $79 \mathrm{pp}$.

AGÜERO, A. (2008): Experiencia de Organización Barrial y Educación en Sectores Populares. "Terceras Jornadas de Historia de la Patagonia". En Navarro Floria, P. (Comp.). Universidad Nacional del Comahue. San Carlos de Bariloche.

ARRIAGADA LUCO, C.; RODRIGUEZ VIGNOLI, J. (2003): Segregación residencial en áreas metropolitanas de América Latina: magnitud, características, evolución e implicaciones de política. Serie Población y Desarrollo N ${ }^{\circ} 47$, CELADE / UNFPA, Santiago de Chile, 72 pp.

BABY-COLLIN, V. (2005): Des marges dans la ville: mobilités citadines et métissage de l'urbanité. "Liens et lieux de la mobilité: ces autres territoires". En Caprón, G.; Cortés, G. y Guetat, H. (Eds.). Coll. Mappemonde. Paris, pp. 145-167.

BAUMAN, Z. (2010): Carajillo: café con picardía. Revista Digital del Programa en Gestión de la Ciudad. "Vivir con extranjeros", año 2, nº 6.

CLICHEVSKY, N. (2000): Informalidad y segregación urbana en América Latina. Una aproximación. CEPAL ECLAC, Santiago de Chile, $24 \mathrm{pp}$.

COUNCIL OF EUROPE (1998): Culture and Neighbourhoods. Council of Europe Publishing. Vol 3, 163 pp.

DIRECCIÓN GENERAL DE ESTADÍSTICA Y CENSOS DE LA PROVINCIA DE RÍO NEGRO (DGEC) (2005): San Carlos de Bariloche por Barrios, Censo Nacional de Población, Hogares y Viviendas 2001. DGEC, Viedma, 1342 pp.

DUHAU, E. (2013): Nueva Sociedad. "La división social del espacio metropolitano. Una propuesta de análisis", n ${ }^{\circ}$ 243, p. 79-91.

FUENTES, R. y NÚÑEZ, P. (2007): Sectores populares: identidad cultural e historia en Bariloche. Editorial Núcleo Patagónico, Bariloche, 201 pp.

GRAFMEYER, Y. (1998): Logement, quartier, sociabilité. "Logement et habitat, l'état des savoirs". En Segaud, M.; Bonvalet, C. y Brun, J. (Eds.). Editorial La Découverte, Coll. textes à l'appui, Paris, pp. 347-354.

GRAVANO, A. (1997): "Variables de lo barrial y lo barrial como variable en la ciudad intermedia". Actas del V Congreso de Antropología Social. La Plata, Argentina.

GRAVANO, A. (2005): El barrio en la Teoría Social. Espacio Editorial, Buenos Aires, 200 pp.

GRIMSON, A. (2009): Articulaciones cambiantes de clase y etnicidad: una villa miseria de Buenos Aires. "La vida política en los barrios populares de Buenos Aires". En Grimson, A.; Ferraudi Curto, M. C. y Segura, R. Prometeo, Buenos Aires, pp. 221-247.

GUEVARA, C. (2014): Revista Argumentos. "Mi barrio es el paraíso, el infierno son los otros. Interacción y fragmentación socio-espacial en Victoria, San Fernando, R.M.B.A. 2014", n $^{\circ}$ 16, p. 98-140.

HARVEY, D. [1977] (2007): Urbanismo y desigualdad social. Séptima Edición, Siglo XXI, Madrid, $340 \mathrm{pp}$.

KROPFF CAUSA, L. (2001): De cómo paisanos y chilotes devienen vecinos. Migración, identidad y estado en San Carlos de Bariloche. Tesis de Licenciatura en Ciencias Antropológicas. Buenos Aires, Argentina. Universidad de Buenos Aires, Facultad de Filosofía y Letras.

LENCIONI, S.; VIDAL-KOPPMANN, S.; HIDALGO, R. Y PEREIRA, P. (org.) (2011): Transformações sócio-territoriais nas metrópoles de Buenos Aires, São Paulo e Santiago. FAUUSP, São Paulo, 304 pp.

MASSEY, D. (1985): New directions in space. "Social relations and spatial structure". En Gregory, J. D. MacMillan, London, pp. 9-19. 
FRAGMENTACIÓN URBANA Y ASOCIACIONES...

MATOSSIAN, B. (2010): Scripta Nova. "Expansión urbana y migración. El caso de los migrantes chilenos en San Carlos de Bariloche como actores destacados en la conformación de barrios populares", vol. XIV, $n^{\circ} 331,76$, p. 1-24.

MATOSSIAN, B. (2012): Migración y segregación urbana en ciudades medias. Chilenos en San Carlos de Bariloche, Patagonia - Argentina. Editorial Académica Española. Berlín, 399 pp.

MEDINA, V. D. (2013): Quid 16. "Rentas urbanas y alternativas de recuperación estatal. Aproximaciones al caso de San Carlos de Bariloche y la participación municipal en la renta diferencial", $n^{\circ} 3$, p. 127-147.

MÉNDEZ, L. (2005): Pueblos y Fronteras de la Patagonia Andina Revista de Ciencias Sociales "Una región y dos ciudades. Puerto Montt y Bariloche; una historia económica compartida", año $5, n^{\circ} 5$, p. 4-11.

MÉNDEZ, L., e IWANOW, W. (2001): Bariloche: las caras del pasado. Manuscritos, Neuquén, 223 pp.

MERKLEN, D. (2005): Pobres ciudadanos. Las clases populares en la era democrática (Argentina, 1983-2003). Editorial Gorla, Buenos Aires, 224 pp.

MUNICIPALIDAD DE SAN CARLOS DE BARILOCHE (2011): Plan de Ordenamiento Territorial, San Carlos de Bariloche, Ambiente Natural. Municipalidad de San Carlos de Bariloche, San Carlos de Bariloche, 14 pp.

NAVARRO FLORIA, P. (2008): La "Suiza argentina", de utopía agraria a postal turística: la resignificación de un espacio entre los siglos XIX y XX. "Terceras Jornadas de Historia de la Patagonia". En Navarro Floria, P. (Coord.). Universidad Nacional del Comahue, Bariloche.

NAVARRO FLORIA, P. y VEJSBJERG, L. (2009): Estudios y perspectivas en turismo. "El proyecto turístico barilochense antes de Bustillo. Entre la prehistoria del Parque Nacional Nahuel Huapi y el desarrollo local", vol. 18, no 4, p. 414-433.

NÚNEZ, P.; MATOSSIAN, B. Y VEJSBJERG, L. (2012): Pasos. Revista de Turismo y Patrimonio Cultural. "Patagonia, de margen exótico a periferia turística. Una mirada sobre un área natural protegida de frontera", vol 10, n 1, p. 47-59.

NÚÑ̃Z, P. G. (2004): Cuadernos del Sur. "Un municipio alejado, una actividad tangencial y los efectos de un profundo cambio institucional: San Carlos de Bariloche 1958 - 1970", no 33, p. 169-188.

OLSSON, J. (2012): Presentación. Venciendo la fragmentación. Para lograr un modelo hermoso. "El camino posible. Producción social del hábitat en América Latina" En Arévalo, M. et al. Ediciones Trilce, Montevideo.

PAVCOVICH, P. (2011): El Barrio. Lo social hecho espacio. Eduvim, Villa María, 172 pp.

PRÉVÔT SCHAPIRA, M. F. (2001): Perfiles Latinoamericanos, Revista de la Sede Académica de México de la Facultad Latinoamericana de Ciencias Sociales. "Fragmentación espacial y social: conceptos y realidades", vol. $9, \mathrm{n}^{\circ} 10$, p. 33-56.

REPÚBLICA ARGENTINA. INSTITUTO NACIONAL DE ESTADÍSTICA Y CENSOS, INDEC (2001): Censo Nacional de Población, Hogares y Viviendas 2001. INDEC, Buenos Aires, http://www.indec.gov.ar/webcenso/index.asp [consulta: 10 de Enero de 2014].

REPÚBLICA ARGENTINA. INSTITUTO NACIONAL DE ESTADÍSTICA Y CENSOS. (2010): Censo Nacional de Población, Hogares y Viviendas 2010. INDEC, Buenos Aires, http://www.censo2010.indec.gov.ar/index.asp [consulta: 10 de Enero de 2014].

SABATINI, F. (2003) La segregación social del espacio en las ciudades de América Latina. División de Programas Sociales, Departamento de Desarrollo Sostenible, Banco Interamericano de Desarrollo, $41 \mathrm{p}$.

SANTOS, M. (1997): A Natureza do Espaço: Técnica e Tempo, Razão e Emoção. $2^{\text {a }}$ edição, Hucitec, São Paulo, 308 pp.

SCHTEINGART, M. (2001): Perfiles Latinoamericanos, Revista de la Sede Académica de México de la Facultad Latinoamericana de Ciencias Sociales. "La división social del espacio en las ciudades", vol. 9, n 10, p. 13-32.

TOPALOV, C. (1984): Ganancias y rentas urbanas, elementos teóricos. Siglo XXI, Madrid, 274 pp. 